Elect. Comm. in Probab. 16 (2011), 706-711

\title{
ABSOLUTE CONTINUITY OF THE LIMITING EIGENVALUE DISTRIBUTION OF THE RANDOM TOEPLITZ MATRIX
}

\author{
ARNAB SEN $^{1}$ \\ Statistical Laboratory, Center for Mathematical Sciences, University of Cambridge, Wilberforce Road, \\ Cambridge, CB3 OWB, UK \\ email: a.sen@statslab.cam.ac.uk \\ BÁLINT VIRÁG \\ Departments of Mathematics and Statistics, University of Toronto, Toronto ON, M5S 2E4, Canada \\ email: balint@math.toronto.edu
}

Submitted September 30, 2011, accepted in final form October 19, 2011

AMS 2000 Subject classification: 35P20, 60B20

Keywords: Toeplitz matrix, eigenvalue distribution, spectral averaging

Abstract

We show that the limiting eigenvalue distribution of random symmetric Toeplitz matrices is absolutely continuous with density bounded by 8 , partially answering a question of Bryc, Dembo and Jiang (2006). The main tool used in the proof is a spectral averaging technique from the theory of random Schrödinger operators. The similar question for Hankel matrices remains open.

\section{Introduction}

An $n \times n$ symmetric random Toeplitz matrix is given by

$$
\mathbf{T}_{n}=\left(\left(a_{|j-k|}\right)\right)_{0 \leq j, k \leq n}
$$

where $\left(a_{j}\right)_{j \geq 0}$ is a sequence of i.i.d. random variables with $\operatorname{Var}\left(a_{0}\right)=1$. For a $m \times m$ Hermitian matrix A, we denote by

$$
\mu(\mathbf{A}):=\frac{1}{m} \sum_{i=1}^{m} \delta_{\lambda_{i}}
$$

the empirical eigenvalue distribution of $\mathbf{A}$, where $\lambda_{j}, 1 \leq j \leq m$ are the eigenvalues of $\mathbf{A}$, counting multiplicity. Bryc, Dembo and Jiang (2006) established using method of moments that with probability $1, \mu\left(n^{-1 / 2} \mathbf{T}_{n}\right)$ converges weakly as $n \rightarrow \infty$ to a nonrandom symmetric probability measure $\gamma$ which does not depend on the distribution of $a_{0}$, and has unbounded support. They conjecture (see Remark 1.1 there) that $\gamma$ has a smooth density. In this note, we give a partial solution:

\footnotetext{
${ }^{1}$ RESEARCH SUPPORTED BY EPSRC GRANT EP/G055068/1.
} 
Theorem 1. The measure $\gamma$ is absolutely continuous with density bounded by 8 .

The actual bound we get is $\frac{16 \sqrt{2}}{\pi}=7.20 \ldots$, but we do not expect it to be optimal.

It seems that the method of moments is of little use in determining the existence of the absolute continuity of the limiting eigenvalue distribution. Indeed our proof goes along a completely different path. We make use of the fact that the spectrum of the Gaussian Toeplitz matrix can be realized as that of some diagonal matrix consisting of independent Gaussians conjugated by an appropriate projection matrix - a fact observed in a recent paper Sen and Virág (2011). The next key ingredient of our proof is a spectral averaging technique (Wegner type estimate) developed by Combes, Hislop and Mourre (1996) in connection to the problem of localization for certain families of random Schrödinger operators.

Our proof does not establish further smoothness property of $\gamma$. The absolute continuity for the limiting distribution of random Hankel matrices also remains open.

\section{Connection between Toeplitz and circulant matrices}

Since $\gamma$ does not depend on the distribution of $a_{0}$, from now on, we will assume, without any loss, that $\left(a_{i}\right)_{i \geq 0}$ are i.i.d. standard Gaussian random variables. The remainder of the section we recall some facts about the connection between Toeplitz matrices and circulant matrices from Sen and Virág (2011). Let $\mathbf{T}_{n}^{\circ}$ be the symmetric Toeplitz matrix which has $\sqrt{2} a_{0}$ on its diagonal instead of $a_{0}$. It can be easily shown (e.g. using Hoffman-Wielandt inequality, see Bhatia (1997)) that this modification has no effect as far as the limiting eigenvalue distribution is concerned.

$\mathbf{T}_{n}^{\circ}$ is the $n \times n$ principal submatrix of a $2 n \times 2 n$ circulant matrix $\mathbf{C}_{2 n}=\left(b_{j-i \bmod 2 n}\right)_{0 \leq i, j \leq 2 n-1}$, where $b_{j}=a_{j}$ for $0<j<n$ and $b_{j}=a_{2 n-j}$ for $n<j<2 n, b_{0}=\sqrt{2} a_{0}, b_{n}=\sqrt{2} a_{n}$. In other words,

$$
\mathbf{Q}_{2 n} \mathbf{C}_{2 n} \mathbf{Q}_{2 n}=\left(\begin{array}{cc}
\mathbf{T}_{n}^{\circ} & \mathbf{0}_{n} \\
\mathbf{0}_{n} & \mathbf{0}_{n}
\end{array}\right), \quad \text { where } \mathbf{Q}_{2 n}=\left(\begin{array}{cc}
\mathbf{I}_{n} & \mathbf{0}_{n} \\
\mathbf{0}_{n} & \mathbf{0}_{n}
\end{array}\right) \text {. }
$$

The circulant matrix can be easily diagonalized as $(2 n)^{-1 / 2} \mathbf{C}_{2 n}=\mathbf{U}_{2 n} \mathbf{D}_{2 n} \mathbf{U}_{2 n}^{*}$ where $\mathbf{U}_{2 n}$ is the discrete Fourier transform, i.e. a unitary matrix given by

$$
\mathbf{U}_{2 n}(j, k)=\frac{1}{\sqrt{2 n}} \exp \left(\frac{2 \pi i j k}{2 n}\right), 0 \leq j, k \leq 2 n-1
$$

and $\mathbf{D}_{2 n}=\operatorname{diag}\left(d_{0}, d_{1}, \ldots, d_{2 n-1}\right)$, where

$$
d_{j}=\frac{1}{\sqrt{2 n}} \sum_{k=0}^{2 n-1} b_{k} \exp \left(\frac{2 \pi i j k}{2 n}\right)=\frac{1}{\sqrt{2 n}}\left[\sqrt{2} a_{0}+(-1)^{n} \sqrt{2} a_{n}+2 \sum_{k=1}^{n-1} a_{k} \cos \left(\frac{2 \pi j k}{2 n}\right)\right] .
$$

Clearly, $d_{j}=d_{2 n-j}$ for all $n<j<2 n$. Also, $\left(d_{j}\right)_{0 \leq j \leq n}$ are independent mean zero Gaussian random variables with $\operatorname{Var}\left(d_{j}\right)=1$ if $0<j<n$ and $\operatorname{Var}\left(d_{j}\right)=2$ if $j \in\{0, n\}$. Define $\mathbf{P}_{2 n}:=\mathbf{U}_{2 n}^{*} \mathbf{Q}_{2 n} \mathbf{U}_{2 n}$ so that

$$
(2 n)^{-1 / 2} \mathbf{U}_{2 n}^{*} \mathbf{Q}_{2 n} \mathbf{C}_{2 n} \mathbf{Q}_{2 n} \mathbf{U}_{2 n}=\mathbf{P}_{2 n} \mathbf{D}_{2 n} \mathbf{P}_{2 n}
$$

Check that $\mathbf{P}_{2 n}$ is a Hermitian projection matrix with $\mathbf{P}_{2 n}(j, j)=1 / 2$ for all $j$. For notational simplification, we will drop the subscript $2 n$ from the relevant matrices unless we want to emphasize the dependence on $n$. 


\section{Proof of the main theorem}

For a vector $\mathbf{u} \in \mathbb{C}^{m}$ and a Hemitian matrix A, let $\sigma(\mathbf{A}, \mathbf{u}):=\sum_{i=1}^{m}\left|\left\langle\mathbf{v}_{i}, \mathbf{u}\right\rangle\right|^{2} \delta_{\lambda_{i}}$ be the spectral measure of $\mathbf{A}$ at $\mathbf{u}$, where $\mathbf{A}=\sum_{i=1}^{m} \lambda_{i} \mathbf{v v}_{i}^{*}$ is a spectral decomposition of $\mathbf{A}$. For a finite measure $v$ on $\mathbb{R}$, its Cauchy-Stieltjes transform is given by

$$
s(z ; v)=\int_{\mathbb{R}} \frac{1}{x-z} v(d x), \quad z \in \mathbb{C}, \operatorname{Im}(z)>0 .
$$

Let $\mathbb{E} \mu\left(n^{-1 / 2} \mathbf{T}_{n}^{\circ}\right)$ denote the expected empirical eigenvalue distribution of $n^{-1 / 2} \mathbf{T}_{n}^{\circ}$ which is defined by $\mathbb{E} \mu\left(n^{-1 / 2} \mathbf{T}_{n}^{\circ}\right)(B)=\mathbb{E}\left[\mu\left(n^{-1 / 2} \mathbf{T}_{n}^{\circ}\right)(B)\right]$ for all Borel sets $B$.

Lemma 2. Let $\left(\mathbf{e}_{j}\right)_{0 \leq j \leq 2 n-1}$ be the coordinate vectors of $\mathbb{R}^{2 n}$. Then

$$
s\left(z ; \mathbb{E} \mu\left(n^{-1 / 2} \mathbf{T}_{n}^{\circ}\right)\right)=\frac{\sqrt{2}}{n} \sum_{j=0}^{2 n-1} \mathbb{E}\left\langle\mathbf{P e}_{j},(\mathbf{P D P}-z \mathbf{I})^{-1} \mathbf{P e}_{j}\right\rangle \quad z \in \mathbb{C}, \operatorname{Im}(z)>0 .
$$

Before we start proving the above lemma, we state a simple fact about spectral measures of Hermitian matrices.

Lemma 3. Let $\mathbf{A}$ be an $m \times m$ Hermitian matrix. Let $\mathbf{u}_{1}, \mathbf{u}_{2}, \ldots, \mathbf{u}_{k}$ and $\mathbf{v}_{1}, \mathbf{v}_{2}, \ldots, \mathbf{v}_{\ell}$ be vectors in $\mathbb{C}^{m}$ satisfying $\sum_{i=1}^{k} \mathbf{u}_{i} \mathbf{u}_{i}^{*}=\sum_{j=1}^{\ell} \mathbf{v}_{j} \mathbf{v}_{j}^{*}$. Then

$$
\sum_{i=1}^{k} \sigma\left(\mathbf{A}, \mathbf{u}_{i}\right)=\sum_{j=1}^{\ell} \sigma\left(\mathbf{A}, \mathbf{v}_{j}\right)
$$

Proof of Lemma 3. Let $\mathbf{A}=\sum_{r=1}^{m} \lambda_{r} \mathbf{w}_{r} \mathbf{w}_{r}^{*}$ be a spectral decomposition of A. Now for each $r$, it follows from the definition of the spectral measure that the probability masses at $\lambda_{r}$ for the both side of (3) are equal. This completes the proof of the lemma.

Proof of Lemma 2. By (1), we have

$$
s\left(z ; \mu\left(n^{-1 / 2} \mathbf{T}_{n}^{\circ}\right)\right)=\frac{1}{n} \sum_{j=0}^{n-1}\left\langle\mathbf{e}_{j},\left(n^{-1 / 2} \mathbf{Q C Q}-z \mathbf{I}\right)^{-1} \mathbf{e}_{j}\right\rangle,
$$

Changing basis as in (2), we can rewrite this as

$$
\frac{\sqrt{2}}{n} \sum_{j=0}^{n-1}\left\langle\mathbf{U}^{*} \mathbf{e}_{j},(\mathbf{P D P}-z \mathbf{I})^{-1} \mathbf{U}^{*} \mathbf{e}_{j}\right\rangle=\frac{\sqrt{2}}{n} \sum_{j=0}^{n-1} s\left(z ; \sigma\left(\mathbf{P D P}, \mathbf{U}^{*} \mathbf{e}_{j}\right)\right) .
$$

Now by Lemma 3 and the fact that $\sum_{j=0}^{n-1} \mathbf{U}^{*} \mathbf{e}_{j} \mathbf{e}_{j}^{*} \mathbf{U}=\sum_{j=0}^{2 n-1} \mathbf{P} \mathbf{e}_{j} \mathbf{e}_{j}^{*} \mathbf{P}$, we deduce

$$
s\left(z ; \mu\left(n^{-1 / 2} \mathbf{T}_{n}^{\circ}\right)\right)=\frac{\sqrt{2}}{n} \sum_{j=0}^{2 n-1}\left\langle\mathbf{P e}_{j},(\mathbf{P D P}-z \mathbf{I})^{-1} \mathbf{P e}_{j}\right\rangle .
$$

The lemma now follows by taking expectation on both sides of (4) and by observing that for a fixed $z \in \mathbb{C}, \operatorname{Im}(z) \neq 0$, the map $v \mapsto s(z ; v)$ is linear and hence commutes with the expectation. 
Next we will prove a key lemma about the uniform bound on the Stieltjes transform of the expected empirical eigenvalue distribution of Toeplitz matrices.

Lemma 4. For all n, we have

$$
\sup _{z: \operatorname{Im}(z)>0}\left|s\left(z ; \mathbb{E} \mu\left(n^{-1 / 2} \mathbf{T}_{n}^{\circ}\right)\right)\right| \leq 16 \sqrt{2} .
$$

The above lemma will be a direct consequence of the following result of Combes et al. (1996) on the spectral averaging for one parameter family self-adjoining operators.

Proposition 5 (Combes et al. (1996)). Let $H_{\lambda}, \lambda \in \mathbb{R}$ be a $C^{2}$-family of self-adjoint operators such that $D\left(H_{\lambda}\right)=D_{0} \subset \mathscr{H} \forall \lambda \in \mathbb{R}$, and such that $\left(H_{\lambda}-z\right)^{-1}$ is twice strongly differentiable in $\lambda$ for all $z, \operatorname{Im}(z) \neq 0$. Assume that there exist a finite positive constant $c_{0}$, and a positive bounded self-adjoint operator B such that, on $D_{0}$

$$
\dot{H}_{\lambda}:=\frac{d H_{\lambda}}{d \lambda} \geq c_{0} B^{2}
$$

Also assume $H_{\lambda}$ is linear in $\lambda$, i.e., $\ddot{H}_{\lambda}:=\frac{d^{2} H_{\lambda}}{d \lambda^{2}}=0$. Then for all $E \in \mathbb{R}$ and twice continuously differentiable function $g$ such that $g, g^{\prime}, g^{\prime \prime} \in L^{1}(\mathbb{R})$ and for all $\varphi \in \mathscr{H}$,

$$
\sup _{\delta>0}\left|\int_{\mathbb{R}} g(\lambda)\left\langle\varphi, B\left(H_{\lambda}-E-i \delta\right)^{-1} B \varphi\right\rangle d \lambda\right| \leq c_{0}^{-1}\left(\|g\|_{1}+\left\|g^{\prime}\right\|_{1}+\left\|g^{\prime \prime}\right\|_{1}\right)\|\varphi\|^{2} .
$$

Proposition 5 is an immediate corollary of Theorem 1.1 of Combes et al. (1996) where instead of $\ddot{H}_{\lambda}=0$, it was assumed that $\left|\ddot{H}_{\lambda}\right| \leq c_{1} \dot{H}_{\lambda}$. The vanishing second derivative assumption shortens the the proof by a considerable amount. We have included a proof of the above proposition in the appendix to make this paper self-contained and also to make constant in the bound (6) explicit.

Proof of Lemma 4. Set $\mathbf{E}_{j}=\mathbf{e}_{j} \mathbf{e}_{j}^{*}+\mathbf{e}_{2 n-j} \mathbf{e}_{2 n-j}^{*}$ for $1 \leq j<n$, and $\mathbf{E}_{j}=\mathbf{e}_{j} \mathbf{e}_{j}^{*}$ for $j \in\{0, n\}$. Take

$$
\mathbf{B}_{j}=\mathbf{P} \mathbf{e}_{j} \mathbf{e}_{j}^{*} \mathbf{P} \text { or } \mathbf{P} \mathbf{e}_{2 n-j} \mathbf{e}_{2 n-j}^{*} \mathbf{P} \text { for } 1 \leq j<n \text { and } \mathbf{B}_{j}=\mathbf{P e}_{j} \mathbf{e}_{j}^{*} \mathbf{P} \text { for } j \in\{0, n\} \text {. }
$$

Fix $0 \leq j \leq n$. We apply Theorem 5 with $H_{\lambda}=\mathbf{P}\left(\mathbf{D}+\left(\lambda-d_{j}\right) \mathbf{E}_{j}\right) \mathbf{P}$. In words, we replace $d_{j}$ and $d_{2 n-j}$ by $\lambda$ in PDP to get $H_{\lambda}$. Note that $H_{\lambda}$ is random self-adjoint operator which is a function of $\left\{d_{k}: 0 \leq k \leq n, k \neq j\right\}$. Also, $H_{\lambda}$ is linear in $\lambda$ and so, $\ddot{H}_{\lambda}=0$. Since $\dot{H}_{\lambda}=\mathbf{P E}_{j} \mathbf{P} \geq \mathbf{B}_{j}=\mathbf{P}(j, j)^{-1} \mathbf{B}_{j}^{2}$, the condition (5) is satisfied with $B=\mathbf{B}_{j}$ and $c_{0}=2$ since $\mathbf{P}(j, j)=1 / 2$. Take $g=\phi_{j}$ where $\phi_{j}$ be the density of $Z$ for $0<j<n$ or the density of $\sqrt{2} Z$ for $j \in\{0, n\}, Z$ being a standard Gaussian random variable. It is easy to check that $\|g\|_{1}=1,\left\|g^{\prime}\right\|_{1} \leq \sqrt{\frac{2}{\pi}},\left\|g^{\prime \prime}\right\|_{1} \leq 2$. Then plugging $\varphi=\mathbf{e}_{j}$ or $\mathbf{e}_{2 n-j}$ and $\mathbf{B}_{j}=\mathbf{P e}_{j} \mathbf{e}_{j}^{*} \mathbf{P}$ or $\mathbf{P} \mathbf{e}_{2 n-j} \mathbf{e}_{2 n-j}^{*} \mathbf{P}$ in (6) and taking expectation w.r.t. the remaining randomness $\left\{d_{k}: 0 \leq k \leq n, k \neq j\right\}$, we obtain

$$
\sup _{z: \operatorname{Im}(z)>0} \mathbf{P}(j, j)^{2}\left|\mathbb{E}\left\langle\mathbf{P e}_{j},(\mathbf{P D P}-z \mathbf{I})^{-1} \mathbf{P} \mathbf{e}_{j}\right\rangle\right| \leq c_{0}^{-1}\left(\|g\|_{1}+\left\|g^{\prime}\right\|_{1}+\left\|g^{\prime \prime}\right\|_{1}\right) \leq 2 .
$$

The lemma is now immediate from (8) and Lemma 2. 
Proof of Theorem 1. From the inversion formula, $v\{(x, y)\}=\lim _{\delta \downarrow} \frac{1}{\pi} \int_{x}^{y} \operatorname{Im}(s(E+i \delta ; v)) d E$ for all $x<y$ continuity points of $v$, it follows that if for some probability measure $\mu, \sup _{z: \operatorname{Im}(z)>0} \operatorname{Im}(s(z ; \mu)) \leq$ $K$ then $\mu$ is absolutely continuous w.r.t. Lebesgue measure and its density is bounded by $\pi^{-1} K$. Note that $s\left(z ; \mathbb{E} \mu\left(n^{-1 / 2} \mathbf{T}_{n}^{\circ}\right)\right) \rightarrow s(z ; \gamma)$ as $n \rightarrow \infty$ for each $z \in \mathbb{C}, \operatorname{Im}(z)>0$ since $\mathbb{E} \mu\left(n^{-1 / 2} \mathbf{T}_{n}^{\circ}\right)$ converges weakly to $\gamma$ (see Bryc et al. (2006)). So by Lemma 4, it follows that

$$
\sup _{z: \operatorname{Im}(z)>0}|s(z ; \gamma)| \leq 16 \sqrt{2}<8 \pi
$$

which completes the proof of the theorem.

\section{Appendix}

Proof of Proposition 5. Define for $\epsilon>0$ and $0<\delta<1$,

$$
R(\lambda, \epsilon, \delta):=\left(H_{\lambda}-E+i \delta+i \epsilon \dot{H}_{\lambda}\right)^{-1}
$$

and set

$$
K(\lambda, \epsilon, \delta):=B R(\lambda, \epsilon, \delta) B
$$

Note that from assumption (5),

$$
-\operatorname{Im}\langle\varphi, K(\lambda, \epsilon, \delta) \varphi\rangle=\left\langle\varphi, B R(\lambda, \epsilon, \delta)^{*}\left(\delta+\epsilon \dot{H}_{\lambda}\right) R(\lambda, \epsilon, \delta) B \varphi\right\rangle \geq c_{0} \epsilon\|K(\lambda, \epsilon, \delta) \varphi\|^{2},
$$

which, coupled with Cauchy-Schwarz inequality, implies that $\forall \varphi \in \mathscr{H},\|\varphi\|=1$,

$$
\|K(\lambda, \epsilon, \delta) \varphi\| \geq-\operatorname{Im}\langle\varphi, K(\lambda, \epsilon, \delta) \varphi\rangle \geq c_{0} \epsilon\|K(\lambda, \epsilon, \delta) \varphi\|^{2} .
$$

Now define

$$
F(\epsilon, \delta):=\int_{\mathbb{R}} g(\lambda)\langle\varphi, K(\lambda, \epsilon, \delta) \varphi\rangle d \lambda .
$$

Inequality (11) implies the bound

$$
F(\epsilon, \delta) \leq\left(\epsilon c_{0}\right)^{-1}\|g\|_{1} .
$$

Now differentiating $F$ w.r.t. $\epsilon$, we obtain

$$
\begin{aligned}
i \frac{d F(\epsilon, \delta)}{d \epsilon} & =\int_{\mathbb{R}} g(\lambda)\left\langle\varphi, B R(\lambda, \epsilon, \delta) \dot{H}_{\lambda} R(\lambda, \epsilon, \delta) B \varphi\right\rangle d \lambda \\
& =-\int_{\mathbb{R}} g(\lambda) \frac{d}{d \lambda}\langle\varphi, K(\lambda, \epsilon, \delta) \varphi\rangle d \lambda .
\end{aligned}
$$

where the last equality follows from the fact $\ddot{H}_{\lambda}=0$. Therefore, from (11) and by integration of parts,

$$
\left|\frac{d F(\epsilon, \delta)}{d \epsilon}\right|=\left|\int_{\mathbb{R}} g^{\prime}(\lambda)\langle\varphi, K(\lambda, \epsilon, \delta) \varphi\rangle d \lambda\right| \leq\left(\epsilon c_{0}\right)^{-1}\left\|g^{\prime}\right\|_{1} .
$$

By integrating the differential inequality (13) and using the bound (12), we can improve the bound for $F$ as

$$
|F(\epsilon, \delta)| \leq c_{0}^{-1}\left\|g^{\prime}\right\|_{1} \cdot|\log \epsilon|+|F(1, \delta)| \leq c_{0}^{-1}\left\|g^{\prime}\right\|_{1} \cdot|\log \epsilon|+c_{0}^{-1}\|g\|_{1}, \quad \forall \epsilon \in(0,1) .
$$


Now if we consider the function $\tilde{F}(\epsilon, \delta):=\int_{\mathbb{R}} g^{\prime}(\lambda)\langle\varphi, K(\lambda, \epsilon, \delta) \varphi\rangle d \lambda$, then by replacing the function $g$ by its derivative $g^{\prime}$ in (14), we deduce that

$$
|\tilde{F}(\epsilon, \delta)| \leq c_{0}^{-1}\left\|g^{\prime \prime}\right\|_{1} \cdot|\log \epsilon|+c_{0}^{-1}\left\|g^{\prime}\right\|_{1}, \quad \forall \epsilon \in(0,1)
$$

which further implies that

$$
\left|\frac{d F(\epsilon, \delta)}{d \epsilon}\right| \leq c_{0}^{-1}\left\|g^{\prime \prime}\right\|_{1} \cdot|\log \epsilon|+c_{0}^{-1}\left\|g^{\prime}\right\|_{1}, \quad \forall \epsilon \in(0,1) .
$$

Again integrating (15), we get

$$
|F(\epsilon, \delta)| \leq c_{0}^{-1}\left(\left\|g^{\prime \prime}\right\|_{1}+\left\|g^{\prime}\right\|_{1}\right)+|F(1, \delta)| \leq c_{0}^{-1}\left(\left\|g^{\prime \prime}\right\|_{1}+\left\|g^{\prime}\right\|_{1}+\|g\|_{1}\right),
$$

which holds for all $\epsilon, \delta \in(0,1)$. The proof of the Proposition now follows from the fact that $R(\lambda, \epsilon, \delta)$ converges weakly to $\left(H_{\lambda}-E+i \delta\right)^{-1}$ as $\epsilon \rightarrow 0+$ provided $\delta>0$, and the dominated convergence theorem since $\left|\int_{\mathbb{R}} g(\lambda)\langle\varphi, K(\lambda, \epsilon, \delta) \varphi\rangle d \lambda\right| \leq C$, by (16).

\section{Acknowledgments}

Most of the research has been conducted while A.S. visited Technical University of Budapest in July 2011.

\section{References}

Bhatia, R. (1997). Matrix analysis, Vol. 169 of Graduate Texts in Mathematics, Springer-Verlag, New York.

Bryc, W., Dembo, A. and Jiang, T. (2006). Spectral measure of large random Hankel, Markov and Toeplitz matrices, Ann. Probab. 34(1): 1-38.

Combes, J. M., Hislop, P. D. and Mourre, E. (1996). Spectral averaging, perturbation of singular spectra, and localization, Trans. Amer. Math. Soc. 348(12): 4883-4894.

Sen, A. and Virág, B. (2011). The top eigenvalue of the random toeplitz matrix and the sine kernel. available at Arxiv math 1109.5494. 